\title{
Diet-induced metabolic hamster model of nonalcoholic fatty liver disease
}

This article was published in the following Dove Press journal:

Diabetes, Metabolic Syndrome and Obesity:Targets and Therapy

2 June 2011

Number of times this article has been viewed

\author{
Jasmine Bhathena \\ Arun Kulamarva \\ Christopher Martoni \\ Aleksandra Malgorzata \\ Urbanska \\ Meenakshi Malhotra \\ Arghya Paul \\ Satya Prakash
}

Biomedical Technology and Cell Therapy Research Laboratory, Department of Biomedical Engineering, Artificial Cells and Organs Research Centre, Faculty of Medicine, McGill University, Montreal, Québec, Canada
Correspondence: Satya Prakash 3775 University Street, Montreal, Québec H3A 2B4, Canada

$\mathrm{Tel}+\mathrm{I} 5 \mathrm{I} 43983676$

Fax + I 514398746 |

Email satya.prakash@mcgill.ca
Background: Obesity, hypercholesterolemia, elevated triglycerides, and type 2 diabetes are major risk factors for metabolic syndrome. Hamsters, unlike rats or mice, respond well to dietinduced obesity, increase body mass and adiposity on group housing, and increase food intake due to social confrontation-induced stress. They have a cardiovascular and hepatic system similar to that of humans, and can thus be a useful model for human pathophysiology.

Methods: Experiments were planned to develop a diet-induced Bio $\mathrm{F}_{1} \mathrm{~B}$ Golden Syrian hamster model of dyslipidemia and associated nonalcoholic fatty liver disease in the metabolic syndrome. Hamsters were fed a normal control diet, a high-fat/high-cholesterol diet, a highfat/high-cholesterol/methionine-deficient/choline-devoid diet, and a high-fat/high-cholesterol/ choline-deficient diet. Serum total cholesterol, high-density lipoprotein cholesterol, low-density lipoprotein cholesterol, triglycerides, glucose, atherogenic index, and body weight were quantified biweekly. Fat deposition in the liver was observed and assessed following lipid staining with hematoxylin and eosin and with oil red $\mathrm{O}$.

Results: In this study, we established a diet-induced Bio $\mathrm{F}_{1} \mathrm{~B}$ Golden Syrian hamster model for studying dyslipidemia and associated nonalcoholic fatty liver disease in the metabolic syndrome. Hyperlipidemia and elevated serum glucose concentrations were induced using this diet. Atherogenic index was elevated, increasing the risk for a cardiovascular event. Histological analysis of liver specimens at the end of four weeks showed increased fat deposition in the liver of animals fed with a high-fat/high cholesterol diet, as compared to animals fed with the control diet.

Conclusion: Our study established that hamsters fed with a high-fat/high-cholesterol diet developed fatty liver and mild diabetes. Bio $\mathrm{F}_{1} \mathrm{~B}$ hamsters fed with a high-fat/high-cholesterol diet may thus be a good animal model for research on the treatment of diet-induced metabolic syndrome complicated by nonalcoholic fatty liver disease.

Keywords: fatty liver disease, in vivo model, diet, atherogenic index, obesity

\section{Introduction}

Obesity is exponentially increasing, and its pervasiveness is at epidemic levels in the world. Obesity may be the cause of or a precursor to other diseases, such as insulin resistance and dyslipidemia (hypertriglyceridemia and reduced high-density lipoprotein [HDL] cholesterol). The term "metabolic syndrome" was coined to describe the concurrent occurrence of these diseases. Individuals with metabolic syndrome are at amplified risk for type 2 diabetes, cardiovascular disease, and nonalcoholic fatty liver disease. ${ }^{1-3}$ The liver is a target organ in metabolic syndrome, in which it manifests itself as nonalcoholic fatty liver disease, spanning the spectrum of hepatosteatosis to hepatocellular carcinoma through steatohepatitis and cirrhosis. Because metabolic 
syndrome and nonalcoholic fatty liver disease affect the same insulin-resistant patients, it stands to reason that there would be a similarity between the metabolic syndrome and nonalcoholic fatty liver disease in terms of prevalence, pathogenesis, clinical features, and outcome. ${ }^{4}$ The costs of treating metabolic syndrome and its associated disorders are growing, and the research community is seeking animal models that mimic the human phenotype so that potential therapies can be tested.

Data from various animal models have provided the conceptual framework for much of the clinical investigations, and permit study of the pathophysiology and fundamental biological mechanisms of disease. Continued studies in animals provide further clarification of the pathogenesis of metabolic disorders and may therefore be very useful to improve diagnosis and treatment of metabolic syndrome. ${ }^{5-7}$ However, the study of the pathophysiologic process of metabolic syndrome and nonalcoholic fatty liver disease is limited by the lack of appropriate animal models that can depict the combined features of nonalcoholic fatty liver disease and the cluster of metabolic abnormalities associated with metabolic syndrome.

Obesity is strongly associated with hepatosteatosis in humans. ${ }^{8}$ Nonetheless, it remains unclear whether the intake of excessive amounts of food by itself causes fatty liver. Because of the pivotal role that diet plays in causing metabolic syndrome in humans, most metabolic disease animal models use diet as a way to precipitate this syndrome. Uncertainty abounds as to whether or not diets that are augmented with certain types of food are more likely to cause obesity and/or fatty liver than other types of diets. It is difficult to control for all of the complex genetic and environmental factors that control energy homeostasis in humans. Therefore, studies that manipulate dietary composition of food and its consumption in animal models may well provide vital insights into the role of diet in the pathogenesis of obesity-related hepatic steatosis.

In order to gain a greater understanding of human obesity, rodents are the commonly used models. Generally, high-fat diets, high sucrose/fructose diets, diets high in saturated fats and restricted in certain essential nutrients, like choline and methionine, have been shown to cause obesity and fatty livers in a number of different strains and species of rodents. ${ }^{9}$ High-fat/high-cholesterol Western diets induce extreme hypercholesterolemia and also lead to concomitant features of the metabolic syndrome, such as weight gain, decreased HDL levels, obesity, hypertriglyceridemia, hyperinsulinemia, and insulin resistance. ${ }^{10-12}$ In addition, these diets generate pathologies independent of atherosclerosis, such as changes in fur and skin integrity, changes in plasma lipids, and hepatic steatosis. ${ }^{13}$ This suggests that "overnutrition" might play a role in the genesis of obesityrelated fatty liver disease and other risk factors associated with metabolic syndrome. ${ }^{14}$ Unfortunately, it is relatively difficult to induce obesity in normal rats and mice. ${ }^{15}$ Not all high-fat diets are the same, because both the level and source of fat may differ between diets. Most rodents tend to become obese on high-fat diets, but there can be variable responses in insulin resistance, triglycerides, and other parameters, depending on the strain and gender, and source of dietary fat. ${ }^{14}$ Normal mice and rats are not ideal models in which to raise the levels of circulating total cholesterol and low-density lipoprotein [LDL] cholesterol, thereby increasing the risk of cardiovascular disease. These models typically have very low levels of total cholesterol and LDL cholesterol, but high levels of HDL cholesterol. ${ }^{14-16}$ This is in contrast to humans, in whom the reverse is true. Moreover, like human populations, rodent populations differ in their susceptibility to diet-induced obesity and fatty liver, suggesting that subtle strain, age, or gender-related variations in genetic factors that regulate intermediary metabolism probably influence the response to various diets. For example, elevated triglycerides are associated with an increase in oxidative stress, and special diets are needed to mimic lipid profiles similar to those of humans. Therefore, it is clear that there is a necessity to develop an animal model of metabolic syndrome expressing fatty liver and other cardiovascular risk factors. To achieve this goal, we used Golden Syrian hamsters, because they have been observed to respond consistently to dietary modulation of cholesterol, and have shown a close similarity to the human lipoprotein profile in comparison with other animals of similar size, eg, rats and mice. ${ }^{17}$

\section{Materials and methods}

\section{Animals}

Male Bio $\mathrm{F}_{1} \mathrm{~B}$ Golden Syrian hamsters (Mesocricetus auratus, 8 weeks old, approximately $90 \mathrm{~g}$ ) were obtained from Biobreeders Inc (Watertown, MA) and housed two per cage in a room with controlled temperature $\left(22-24^{\circ} \mathrm{C}\right)$, humidity and an inverse alternating light and dark cycle (12:12-hour light:dark cycle, lights on at $7 \mathrm{pm}$ ). All experimental protocols complied with the Animal Care Committee of McGill University and Canadian Council on Animal Care guidelines. 


\section{Experimental protocol and diets}

After arrival, animals were allowed free access to a basal diet of commercial rodent ration (LabDiet ${ }^{\circledR}$ rodent laboratory chow 5001, Purina Laboratories, St Louis, MO) and water for two weeks to allow adaptation to the environment. Baseline values of serum total cholesterol were measured at the end of two weeks in hamsters that were deprived of food overnight. Further, these basal serum total cholesterol values were used to assign animals into four groups using a randomized block design. The control group continued to be fed the reference diet 5001. Each of the other groups $(\mathrm{n}=8)$ was from then onwards fed a grain-based, hypercholesterolemic chow diet (Modified LabDiet laboratory rodent diets with added cholesterol and 6\% coconut oil as saturated fat, Purina Laboratories, see Table 1), with free access to water for five weeks. Diet consumption and body weight were measured every 10 days. Food efficiency ratio was computed as g body weight gain/g feed intake. Blood samples were collected once every 14 days from food-deprived hamsters (14 hours) that has been mildly sedated using $3 \mu \mathrm{L}$ of $5 \mathrm{mg} / \mathrm{mL}$ acepromazine. Briefly, after immobilizing the hamster, approximately $150 \mu \mathrm{L}$ of blood was collected from the lateral saphenous vein which runs dorsally and then laterally over the tarsal joint with a sterile 23 gauge $/ 25 \mathrm{~mm}$ needle, into Microtainer ${ }^{\mathbb{B}}$ serum separator tubes from Becton Dickinson (Franklin Lakes, NJ). At the end of the experimental period (five weeks), the hamsters were euthanized by carbon dioxide asphyxiation and blood was withdrawn by cardiac puncture using a 22 gauge $/ 25 \mathrm{~mm}$ needle and a $5 \mathrm{~mL}$ syringe. Cardiac blood was transferred into serum separator tubes and allowed to clot prior to placement on ice.

\section{Serum cholesterol and triglyceride measurements}

Blood from hamsters that had been food deprived for 14 hours was collected under mild sedation into serum separator tubes. The blood was allowed to clot at $23^{\circ} \mathrm{C}$ for 30 minutes and subsequently placed at $4^{\circ} \mathrm{C}$ until centrifugation. Serum was separated by low-speed centrifugation at $2000 \mathrm{~g}$ for 20 minutes at $4{ }^{\circ} \mathrm{C}$ temperature. Serum was frozen at $-85^{\circ} \mathrm{C}$ until analysis for serum total cholesterol, HDL cholesterol, LDL cholesterol, triglycerides, and glucose. Serum total lipids and glucose were assayed by conventional enzymatic methods on a Hitachi 911 automated analyzer from Roche Diagnostics (Laval, QC, Canada). Total cholesterol, HDL cholesterol, triglycerides, and glucose were measured on the Hitachi 911 automated analyzer using reagent kits supplied by Roche Diagnostics. The precision performance of these assays was within the manufacturer's specifications. LDL

Table I Profile of the normal and hypercholesterolemic test diets

\begin{tabular}{|c|c|c|c|c|}
\hline \multirow[b]{2}{*}{ Nutritional profile } & \multirow{2}{*}{$\begin{array}{l}\text { LabDiet }^{\circledR} \text { reference } \\
\text { rodent laboratory chow } \\
5001\end{array}$} & \multicolumn{3}{|c|}{ Modified LabDiet laboratory rodent test diet } \\
\hline & & $5 A 4 C$ & $5 \mathrm{D} 4 \mathrm{~F}$ & 5D4E \\
\hline Protein, \% & 23.9 & 25 & 15.1 & 22.5 \\
\hline Fat (ether extract), \% & 5 & 10.3 & 10 & 10.2 \\
\hline Total saturated fatty acids, $\%$ & 1.56 & 6 & 6 & 5.89 \\
\hline Total monounsaturated fatty acids, $\%$ & 1.6 & 2.10 & 1.24 & 2.10 \\
\hline Polyunsaturated fatty acids, $\%$ & 1.42 & 1.15 & 2.19 & 0.87 \\
\hline Cholesterol, ppm & 200 & 500 & 500 & 500 \\
\hline Linoleic acid, \% & 1.22 & 1.57 & 2.16 & 1.60 \\
\hline Omega-3 fatty acid, \% & 0.19 & 0.19 & 0.03 & 0.14 \\
\hline Choline chloride, ppm & 2250 & 2299 & 0.00 & 1200 \\
\hline Methionine, \% & 0.67 & 0.43 & 0.13 & 0.4 \\
\hline Fiber, \% & 5.1 & 5.1 & 0.0 & 4.6 \\
\hline Nitrogen-free extract, \% & 48.7 & 42.5 & - & 46.4 \\
\hline Starch, \% & 31.9 & 28.37 & - & 36.89 \\
\hline Sucrose, \% & 3.70 & 2.21 & - & 1.34 \\
\hline Total digestible nutrients, \% & 76 & 81 & - & 80.9 \\
\hline \multicolumn{5}{|l|}{ Calories provided by: } \\
\hline Protein, \% & 28.5 & 27.5 & 14.4 & 24.5 \\
\hline Fat, \% & 13.5 & 25.6 & 21.3 & 25 \\
\hline Carbohydrates, $\%$ & 57.9 & 46.9 & 64.3 & 50.6 \\
\hline
\end{tabular}

Abbreviations: 5A4C, diet adequate in methionine and choline levels; 5D4F, diet deficient in methionine and devoid of choline; 5D4E, diet deficient in choline but with adequate levels of methionine. 
cholesterol was calculated by the Friedewald equation. ${ }^{18}$ The atherogenic index was determined as (total cholesterolHDL cholesterol)/HDL cholesterol. ${ }^{19}$

\section{Collection of the liver}

The whole liver was excised from each animal, immersed in chilled phosphate-buffered saline and blotted dry. A $4 \mathrm{~mm}$ section of the liver was placed into a histological cassette. The cassette containing the liver section of each animal was individually immersed in a 10\% (v/v) buffered formalin phosphate solution for fixing and subsequent staining.

\section{Histology}

Liver sections soaked in $10 \%$ buffered formalin phosphate solution were processed for normal histological section. The formalin-fixed, paraffin-embedded tissue samples were ultrasectioned (4-5 $\mu \mathrm{m}$ thickness), stained with hematoxylin and eosin and Oil red $\mathrm{O}$, and examined under a light microscope.

\section{Statistical analysis}

Results were expressed as mean \pm standard deviation. The significance of the difference between the means of test and control studies was established by repeated-measures analysis of variance. An alpha level of 0.05 was set to determine statistical significance $(P<0.05)$.

\section{Results}

Following a two-week acclimatization to the basal control diet, the animals were sorted into control and diet groups based on basal serum total cholesterol concentrations to start the experimental period. During the four-week experimental acute feeding period, the animals were divided into four groups, including control animals that received a normal diet. Three different hypercholesterolemic-hyperlipidemic diets were tested during the study, ie, a diet with adequate methionine and choline levels (5A4C), a diet deficient in choline but with adequate levels of methionine (5D4E) and, lastly, a diet which was deficient in methionine and devoid of choline (5D4F).

\section{Body weight and general health}

The body weights of all animals were monitored at 10-day intervals and are presented in Figure 1. Animals in all treatment groups showed an increase in body weight over the duration of study, which could be attributed to the normal growth phase and the hyperlipidemic diets. The animals fed on the 5D4E diet showed the highest increase in body weight $(21.3 \%)$, with each hamster putting on $7.25 \mathrm{~g}$ per week. The $5 \mathrm{~A} 4 \mathrm{C}$ diet induced a $16 \%$ increase in body weight over the four-week period, with each animal gaining an average of $5.25 \mathrm{~g}$ per week. The normal diet leads to an $8.6 \%$ increase in body weight, corresponding to a weight gain of $2.75 \mathrm{~g}$ per animal per week. In contrast, hamsters fed on the 5D4F diet did not show any significant weight gain during the experimental period. Diets deficient in methionine and choline have been proven to lead to weight loss in rodent models studied earlier. ${ }^{20}$ However, the hamsters fed on this choline-devoid diet showed marked differences in their physical appearance. These animals showed crusting of the upper lip, loss of fur texture with the hair appearing wet or greasy, and were generally very sluggish. The animals fed on the other diets were all very active and appeared to be in good health. Food intake was significantly lower in hamsters on diets which were either

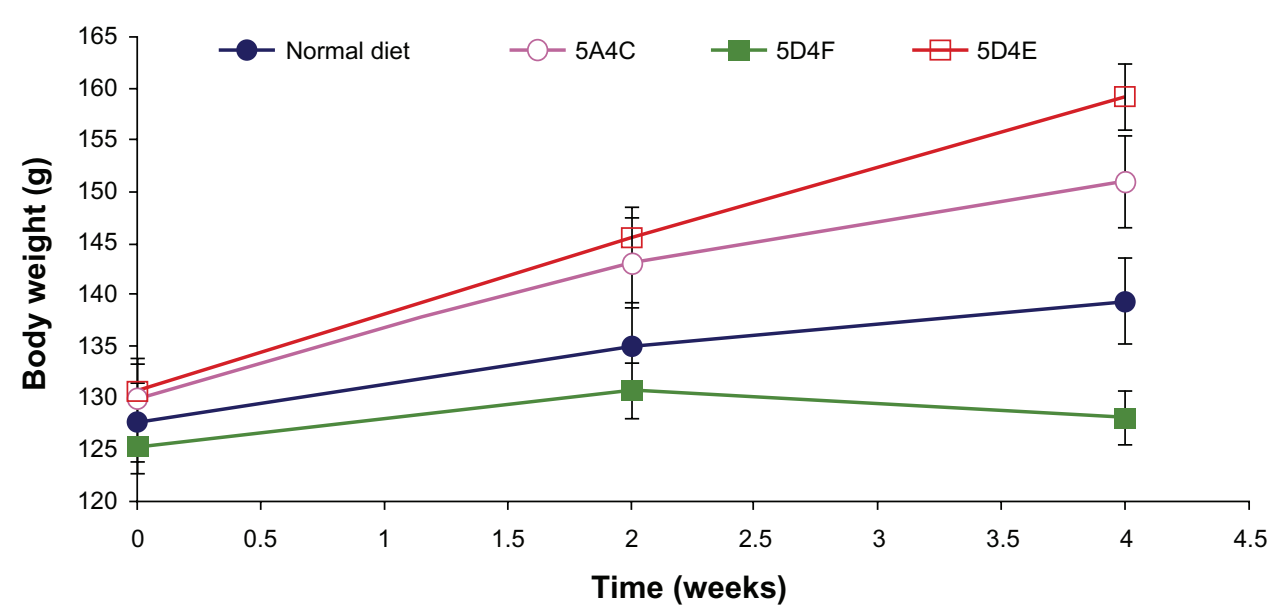

Figure I Average animal weight on food intake and food efficiency ratio. A) normal diet, B) 5A4C, C) 5D4F, and D) 5D4E.

Abbreviations: $5 \mathrm{~A} 4 \mathrm{C}$, diet adequate in methionine and choline; 5D4F, diet deficient in methionine and choline; 5D4E, diet adequate in methionine and deficient in choline. 
deficient or devoid of choline (Figure 1) compared with the other two diets. Methionine-deficient and choline-deficient diets have been associated with lower food intakes in prior studies on rodents. ${ }^{21,22}$ The 5D4E diet showed the best food efficiency ratio among the diets evaluated.

\section{Serum total cholesterol levels}

Compared with the baseline control level, serum total cholesterol levels were elevated in all groups of animals on the test diets (Figure 2A). As other short-term studies have shown, this elevation of serum total cholesterol concentration likely resulted from the dietary cholesterol ingested. ${ }^{23,24}$ Hamsters fed the 5D4E diet showed a dramatic increase $(232 \%)$ in serum total cholesterol levels $(P<0.0001)$. The 5A4C diet led to a $25 \%$ increase in total cholesterol levels over the fourweek study $(P=0.084)$. Total cholesterol levels in hamsters on the 5D4F diet increased during the first two weeks, but reduced during the next two weeks, showing a $16 \%$ reduction in serum total cholesterol levels $(P=0.038)$.

\section{A}

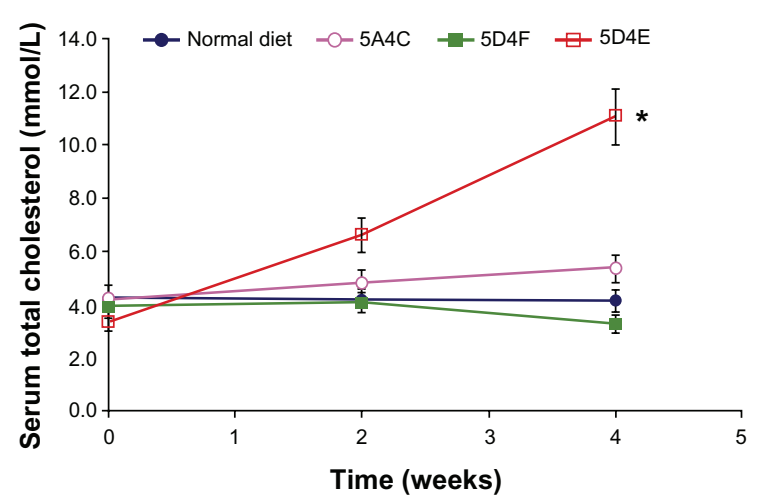

C

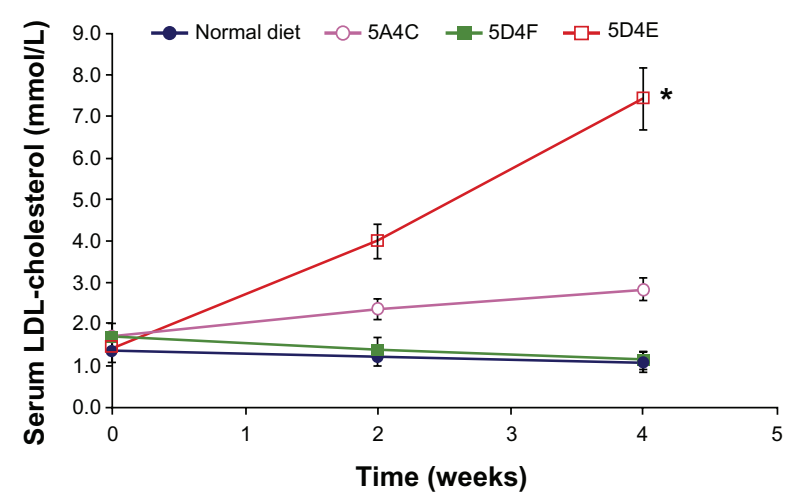

\section{Serum HDL and LDL cholesterol} and triglycerides

HDL cholesterol decreased over the study period in all the experimental diet groups compared with the group on the normal diet (Figure 2B). The 5D4F diet showed the highest decrease in serum HDL cholesterol levels $(P<0.0001)$, followed by the 5D4E $(P=0.0006)$ and 5A4C $(P=0.0014)$ diets. In contrast, hamsters fed with the 5D4E diet showed a dramatic increase in LDL cholesterol levels $(P<0.0001)$ during the study, compared with those on a normal diet, the values for which remained stable (Figure $2 \mathrm{C}$ ). The 5A4C diet had a similar effect on LDL cholesterol levels $(P=0.0049)$ although not to the same extent as did the 5D4E diet. Serum LDL cholesterol values for animals fed with the 5D4F diet showed a decreasing trend $(P=0.82)$. Hamsters fed with the 5D4E diet $(P=0.0007)$, 5D4F diet $(P=0.27)$, and the normal diet showed an increase in serum triglycerides, while the triglyceride values decreased for those with the $5 \mathrm{~A} 4 \mathrm{C}$ $\operatorname{diet}(P=0.086$, Figure 2D).
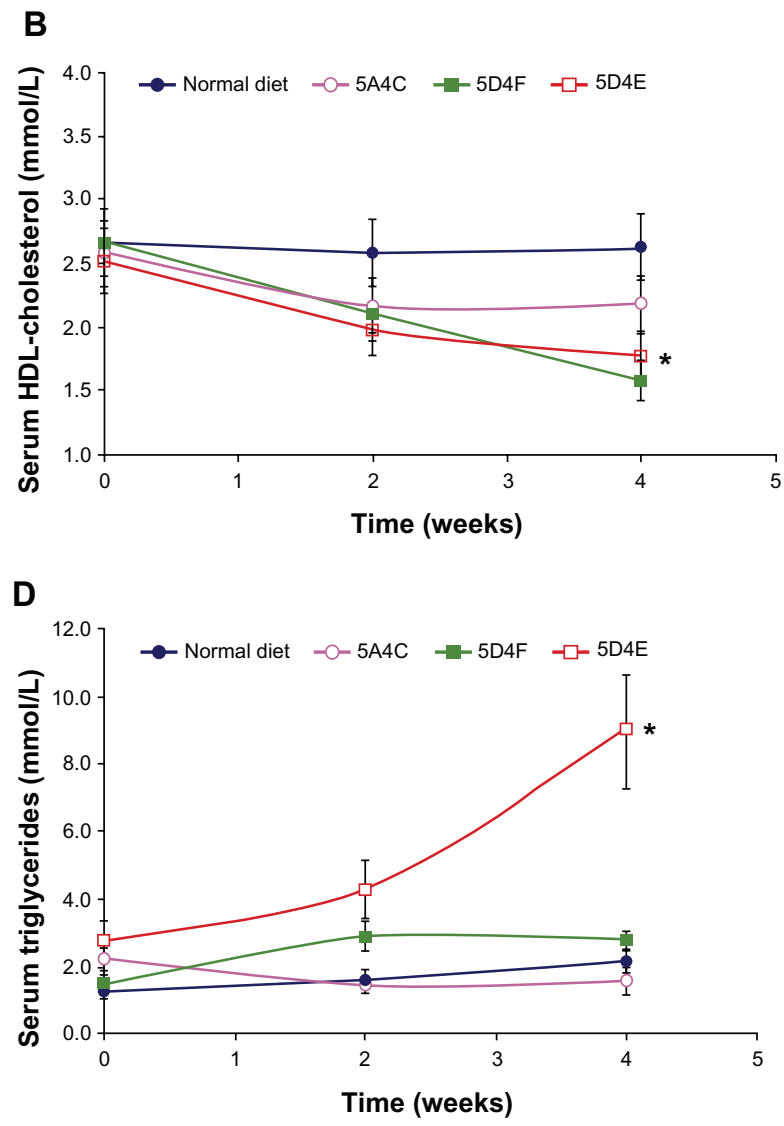

Figure 2 Changes with time in serum A) total cholesterol, B) HDL cholesterol, C) LDL cholesterol, and D) triglycerides of hamsters ( $\mathrm{n}=8$ per group) fed with a normal diet, 5A4C, 5D4F, or 5D4E. Animals were sacrificed after having been fed the respective diets ad libitum for four weeks. Liver triglycerides and serum lipoproteins were determined biweekly. Each point represents the mean \pm standard deviation.

Notes: $* P<0.000$ I for total cholesterol; $* P=0.0006$ for HDL cholesterol; $* P<0.000$ I for LDL cholesterol; and $* P=0.0007$ for triglycerides.

Abbreviations: HDL, high-density lipoprotein; LDL, low-density lipoprotein; 5A4C, diet adequate in methionine and choline; 5D4F, diet deficient in methionine and choline; 5D4E, diet adequate in methionine and deficient in choline. 


\section{Glucose and atherogenic index}

Glucose levels were elevated in all animals to varying extents. These doubled in the hamsters fed on the 5D4E $\operatorname{diet}(P<0.0001)$, while those on the 5A4C diet showed an increase of $85 \%$ compared with baseline values $(P=0.011)$. In comparison, serum glucose levels showed a $48 \%$ and $25 \%$ increase in hamsters fed with the $5 \mathrm{D} 4 \mathrm{~F} \operatorname{diet}(P=0.0046)$ and normal diets, respectively (Figure 3A). The atherogenic index, which is correlated with cardiovascular disease, increased over five-fold in animals on the 5D4E diet $(P<0.0001)$, and by $50 \%$ in animals on the $5 \mathrm{~A} 4 \mathrm{C} \operatorname{diet}(P=0.0008)$. It remained stable in animals on the normal and 5D4F diets $(P=0.0020$, Figure 3B)

\section{Histopathology}

Histopathological analysis of hamster liver samples through $\mathrm{H} \& \mathrm{E}$ and Oil red $\mathrm{O}$ staining show marked differences between the diets studied. Hematoxylin and eosin staining demonstrated elevated amounts of fat deposits in liver tissue from animals fed on the test diets (Figures 4B, 4C, and 4D) compared with the normal diet (Figure 4A). Macrovesicular deposition of fat and hepatocellular ballooning was observed in hamsters fed on the 5D4F (Figure 4B) while microvesicular fat deposits and ballooning to a lesser extent were found in hamsters on diets of 5A4C (Figure 4C) and 5D4E (Figure 4D). Oil red O staining of the liver tissue samples substantiate these results (Figure 5). A very high amount
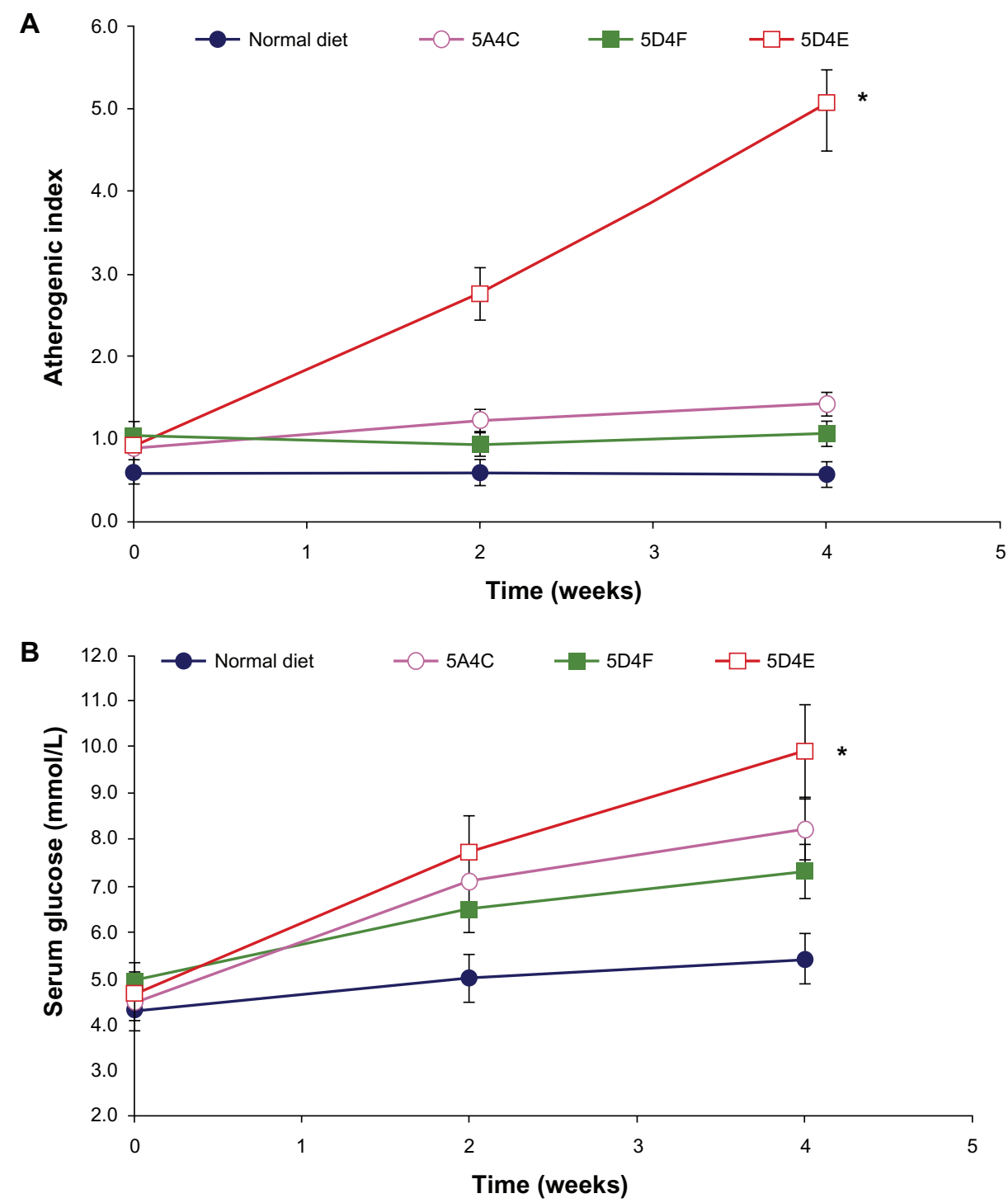

Figure 3 Effect on $\mathbf{A}$ ) atherogenic index and $\mathbf{B}$ ) serum glucose on Bio $F_{1} B$ hamsters ( $n=8$ per group) on administering a normal diet, 5A4C, 5D4F, or 5D4E. Each point represents the mean \pm standard deviation.

Notes: $* P<0.000$ I for serum glucose and atherogenic index.

Abbreviations: 5A4C, diet adequate in methionine and choline; 5D4F, diet deficient in methionine and choline; 5D4E, diet adequate in methionine and deficient in choline. 

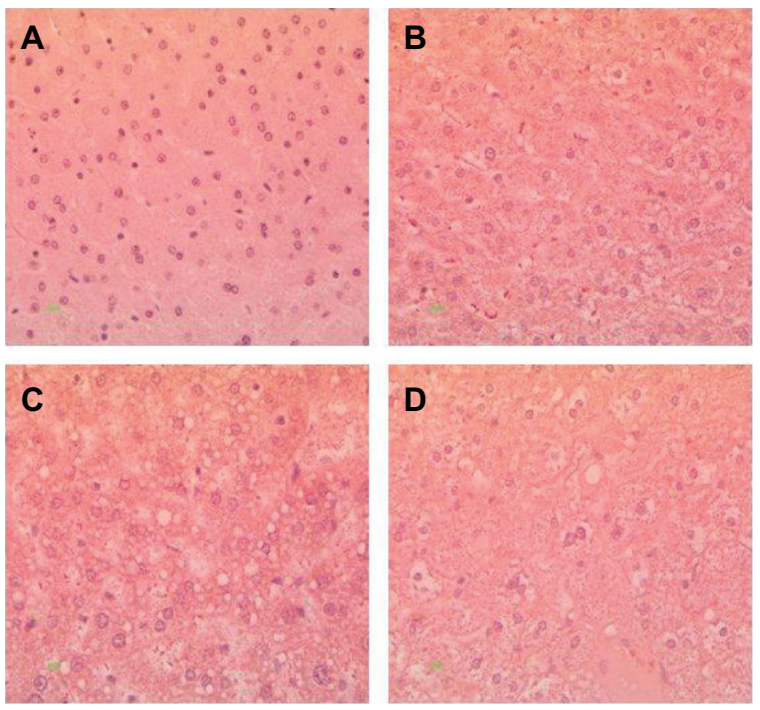

Figure 4 Hematoxylin and eosin staining of liver tissue from hamsters fed with hyperlipidemic diets. Magnification 400x. A) Normal diet, B) diet adequate in methionine and choline (5A4C), C) diet deficient in methionine and choline (5D4F), and D) diet adequate in methionine and deficient in choline (5D4E). Hepatocytes are filled with microvascular and macrovesicular fat deposits, leaving the nuclei in a central position, and the hepatocytes have assumed a very foamy appearance.

of fat deposition was found in hamsters fed on diet 5D4F (Figure 5C), followed by 5D4E (Figure 5D). Histological analysis confirm the development of fatty liver in animal models fed with the three test diets, compared with those on a normal diet. However, there were no signs of fibrosis.

\section{Discussion}

Animal models offer a convenient medium to investigate and understand the pathophysiology of disease and facilitate
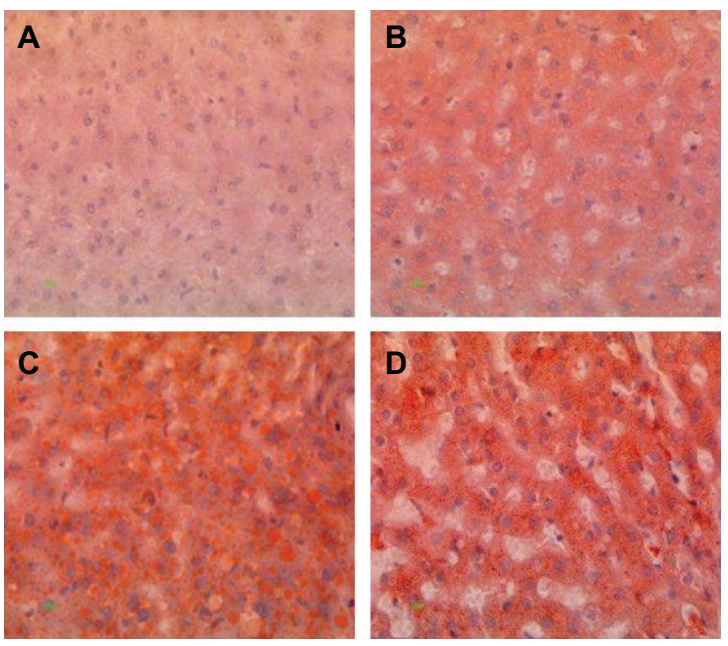

Figure 5 Oil red $\mathrm{O}$ staining of liver tissue from hamsters fed on hyperlipidemic diets. Magnification $400 \times$. A) Normal diet, B) diet adequate in methionine and choline (5A4C), C) diet deficient in methionine and choline (5D4F), and D) diet adequate in methionine and deficient in choline (5D4E). Hamster hepatocytes are filled with microvesicular and/or macrovesicular fat deposits; they are depicted as reddish-orange deposits, as shown with Oil red $O$ staining. development of means to prevent or treat the studied disease. Studies on such models greatly contribute towards enhancing knowledge in the field. Animal models that express clinical manifestations of metabolic syndrome, such as insulin resistance, fatty liver, and dyslipidemia, will be of immense value in understanding metabolic syndrome. We have investigated induction of metabolic syndrome in male Bio $\mathrm{F}_{1} \mathrm{~B}$ Golden Syrian hamsters through nutritional intervention. The use of only male hamsters was encouraged to avoid gender-related response due to female hormones on a fatty acid diet. ${ }^{25}$

Serological and histopathological changes in hamsters fed on hyperlipidemic diets varying in methionine and choline levels in comparison with a normal diet were evaluated during this study. Our results suggest that BioF1B Golden Syrian hamsters are a potential model for diet-induced metabolic syndrome with associated non-alcoholic fatty liver disease.

Among the three test diets, the choline-deficient 5D4E diet provided optimal induction of metabolic syndrome, while the methionine-deficient/choline-devoid 5D4F lead to some undesirable effects on the normal well being of the animals. The hamsters displayed several manifestations of the human metabolic syndrome. The hyperlipidemic effects of the diets were visibly demonstrated in the increased body weight and lipid profile of serological samples from hamsters fed on the test diets. The animals showed hyperglycemia and an elevated atherogenic index, which are commonly associated with metabolic syndrome. In addition, histopathological analysis revealed extensive diet-induced hepatocellular fat deposition and ballooning in the liver samples from the hamsters, while the control hamsters had normal liver histology. However, the spectrum of conditions characterized by fatty change in the liver had not progressed to necrosis, fibrosis, or inflammation. In comparison with the normal diet, the hyperlipidemic diets were also associated with elevated serum total cholesterol levels, decreased HDL cholesterol, and hyperglycemia and hypertriglyceridemia, thereby confirming the induction of metabolic syndrome and nonalcoholic fatty liver in the hamsters.

It is evident that special diets are needed to develop and study an animal model of metabolic syndrome. However, no phenotype of any animal model is guaranteed, and cautious choosing of the species and strain as well as satisfactory control over environmental factors is important. In this paper, we have shown that BioF1B Golden Syrian hamsters can be used as a model that develops clinical and histopathological manifestations of the human metabolic syndrome through dietary intervention. It is known that the dietary factors may promote multiple phenotypes, ${ }^{26,27}$ for example, the use 
of high-fat diets induces obesity, insulin resistance, and hyperglycemia and the use of high-fructose diets promote insulin resistance, hypertriglyceridemia, and hypertension. However, we did not monitor for reversion of the phenotypic characteristics of the animal model by replacing their diet from hyperglycemic to normal diet. In addition, it should be noted that the hamster diet, although adequate for the proposed work, does not represent an actual human diet that can potentially stimulate nonalcoholic fatty liver disease. Thus, this study opens up new approaches to investigate further the long-term effects of choline deprivation on the animal model. This concurrent advance of diseases is not unexpected, given the multifaceted interactions and relationships between these diseases. At present, diet-induced animal models of metabolic syndrome are still being developed, and there may not be one single model that will satisfy all metabolic disease research needs. Ongoing research using different species/strains along with existing and new purified ingredient diet formulations should lead to the development of more and more useful metabolic syndrome phenotypes.

\section{Conclusion}

In conclusion, we have developed a successful model of metabolic syndrome in BioF1B Golden Syrian hamsters. This metabolic syndrome model with hyperlipidemia and insulin resistance was established (along with nonalcoholic fatty liver) in hamsters fed a high-fat, high-cholesterol, inadequate methionine- and choline-containing diet. This model may be useful for the evaluation of preventive medicine, including food factors, for obesity-induced metabolic syndrome. The increasing prevalence of obesity, diabetes and insulin resistance, and nonalcoholic fatty liver disease within Western society makes research in this field vital. In addition, using this model, it may be possible to elucidate the mechanisms involved in the development of metabolic syndrome, especially the association between lipid accumulation-induced dysfunction of hepatocytes and the induction of insulin resistance. It is only through better understanding of pathogenic mechanisms that novel therapies targeting the cluster of diseases in metabolic syndrome may be discovered.

\section{Acknowledgment}

The authors acknowledge the support of a Canadian Institutes of Health Research (CIHR; MOP-94308) Micropharma research contract grant (to SP). A doctoral research award from the Canadian Institutes of Health Research to JB, a Canada graduate scholarship from NSERC to CM, an Alexander Graham Bell Canada graduate scholarship from
NSERC to AK, a McGill University majors scholarship to $\mathrm{MM}$, and a postgraduate scholarship from NSERC to AMU. AP is grateful for the financial support from a NSERC Alexander Graham Bell Canada graduate scholarship. We would also like to acknowledge the technical support of Melina Narlis.

\section{Disclosure}

SP and JP have a conflict of interest with Micropharma Ltd, given that the technology mentioned in this research is optioned to Micropharma Ltd. None of the other authors have any personal or financial conflicts of interest to report in this work.

\section{References}

1. Eckel RH, Grundy SM, Zimmet PZ. The metabolic syndrome. Lancet. 2005;365:1415-1428.

2. Alberti KGMM, Zimmet P, Shaw J. The metabolic syndrome - a new worldwide definition. Lancet. 2005;366:1059-1062.

3. Grundy SM, Cleeman JI, Daniels SR, et al. Diagnosis and management of the metabolic syndrome - An American Heart Association/National Heart, Lung, and Blood Institute Scientific Statement. Circulation. 2005; 112:2735-2752.

4. Khashab MA, Liangpunsakul S, Chalasani N. Nonalcoholic fatty liver disease as a component of the metabolic syndrome. Curr Gastroenterol Rep. 2008;10:73-80.

5. Tarling EJ, Ryan KJ, Bennett AJ, et al. Effect of dietary conjugated linoleic acid isomers on lipid metabolism in hamsters fed high-carbohydrate and high-fat diets. Br J Nutr. 2009;101:1630-1638.

6. Basciano H, Miller AE, Naples M, et al. Metabolic effects of dietary cholesterol in an animal model of insulin resistance and hepatic steatosis. Am J Physiol Endocrinol Metab. 2009;297:E462-E473.

7. Gao S, He L, Ding Y, Liu G. Mechanisms underlying different responses of plasma triglyceride to high-fat diets in hamsters and mice: Roles of hepatic MTP and triglyceride secretion. Biochem Biophys Res Commun. 2010;398:619-626.

8. Ratziu V, Giral P, Charlotte F, et al. Liver fibrosis in overweight patients. Gastroenterology. 2000;118:1117-1123.

9. Koteish A, Diehl AM. Animal models of steatosis. Semin Liver Dis. 2001;21:89-104.

10. Hartvigsen K, Binder CJ, Hansen LF, et al. A diet-induced hypercholesterolemic murine model to study atherogenesis without obesity and metabolic syndrome. Arterioscler Thromb Vasc Biol. 2007;27:878-885.

11. Schreyer SA, Vick C, Lystig TC, Mystkowski P, LeBoeuf RC. LDL receptor but not apolipoprotein $\mathrm{E}$ deficiency increases diet-induced obesity and diabetes in mice. Am J Physiol Endocrinol Metab. 2002;282: E207-E214.

12. Li AC, Brown KK, Silvestre MJ, Willson TM, Palinski W, Glass CK. Peroxisome proliferator-activate inhibit development of atherosclerosis in LDL receptor-deficient mice. J Clin Invest. 2000;106:523-531.

13. Lichtman AH, Clinton SK, Iiyama K, Connelly PW, Libby P, Cybulsky MI. Hyperlipidemia and atherosclerotic lesion development in LDL receptor-deficient mice fed defined semipurified diets with and without cholate. Arterioscler Thromb Vasc Biol. 1999;19:1938-1944.

14. Gajda A, Pellizzon M, Ricci M, Ulman E. Diet-induced metabolic syndrome in rodent models. Animal Lab News. March 2007. www. researchdiets.com/OSD/DIDM/metabolic.htm. Accessed April 21, 2011.

15. Harrold JA, Widdowson PS, Clapham JC, Williams G. Individual severity of dietary obesity in unselected Wistar rats: Relationship with hyperphagia. Am J Physiol Endocrinol Metab. 2000;279:E340-E347. 
16. Maxwell KN, Soccio RE, Duncan EM, Schayek E, Breslow JL. Novel putative SREBP and LXR target genes identified by microarray analysis in liver of cholesterol-fed mice. J Lipid Res. 2003;44: 2109-2119.

17. Bravo E, Cantafora A, Calcabrini A, Ortu G. Why prefer the Golden Syrian-hamster (Mesocricetus auratus) to the Wistar rat in experimental studies on plasma-lipoprotein metabolism. Comp Biochem Physiol B Biochem Mol Biol. 1994;107:347-355.

18. Friedawald W, Levy RI, Fredrickson DS. Estimation of concentration of low-density lipoprotein cholesterol in plasma, without use of preparative ultracentrifuge. Clin Chem. 1972;18:499-502.

19. Matsubara M, Maruoka S, Katayose S. Decreased plasma adiponectin concentrations in women with dyslipidemia. J Clin Endocrinol Metab. 2002;87:2764-2769.

20. Rizki G, Arnaboldi L, Gabrielli B, et al. Mice fed a lipogenic methioninecholine-deficient diet develop hypermetabolism coincident with hepatic suppression of SCD-1. J Lipid Res. 2006;47:2280-2290.

21. Romestaing C, Piquet MA, Bedu E, et al. Long term highly saturated fat diet does not induce NASH in Wistar rats. Nutr Metab (Lond). 2007:4.
22. Gorustovich AA, Esposito MA, Guglielmotti MB, Giglio MJ. Mandibular bone remodeling under a choline-deficient diet: A histomorphometric study in rats. J Periodontol. 2003;74:831-837.

23. Dorfman SE, Smith DE, Osgood DP, Lichtenstein AH. Study of diet-induced changes in lipoprotein metabolism in two strains of Golden-Syrian hamsters. J Nutr. 2003;133:4183-4188.

24. Kahlon TS, Chow FI, Irving DW, Sayre RN. Cholesterol response and fatty streak formation in hamsters fed two levels of saturated fat and various levels of cholesterol. Nutr Res. 1997;17:1693-1707.

25. Morise A, Mourot J, Boué C, et al. Gender-related response of lipid metabolism to dietary fatty acids in the hamster. Br J Nutr. 2006;95: 709-720.

26. Kim HJ, Jeon SM, Lee MK, et al. Comparison of hesperetin and its metabolites for cholesterol-lowering and antioxidative efficacy in hypercholesterolemic hamsters. J Med Food. 2010;13:808-814.

27. Liao FH, Liou TH, Shieh MJ, et al. Effects of different ratios of monounsaturated and polyunsaturated fatty acids to saturated fatty acids on regulating body fat deposition in hamsters. Nutrition. 2010;26: 811-817.

\section{Publish your work in this journal}

Diabetes, Metabolic Syndrome and Obesity: Targets and Therapy is an international, peer-reviewed open-access journal committed to the rapid publication of the latest laboratory and clinical findings in the fields of diabetes, metabolic syndrome and obesity research. Original research, review, case reports, hypothesis formation, expert opinion and commentaries are all considered for publication. The manuscript management system is completely online and includes a very quick and fair peer-review system, which is all easy to use. Visit http://www.dovepress.com/testimonials.php to read real quotes from published authors.

Submit your manuscript here: http://www.dovepress.com/diabetes-metabolic-syndrome-and-obesity-targets-and-therapy-journal 